\title{
PALLIATIVE CARE FOR NEWBORN INFANTS - THE CURRENT SCENE IN NEW ZEALAND AND THE WAY FORWARD
}

\author{
Vazhkudai Sankaran Kumaran \& Yvonne Bray
}

\begin{abstract}
Palliative care is increasingly recognized as an important aspect of neonatal care of medical illness especially with neonatal deaths accounting for half the children dying under five years of age. This article examines the state of palliative care for newborn infants in New Zealand and discusses the issues involved in the organization of a comprehensive neonatal palliative care strategy.
\end{abstract}

\section{INTRODUCTION}

The World Health Organization defines palliative care for children as an 'active total care of the body, mind and spirit of the child and his/her family' (wHo Palliative Care 1998). For a new born baby faced with a life threatening illness the time to deliberate on all these dimensions is very limited. Neonatal deaths still contribute to three-fifths of infant deaths and to about half of under- 5 mortality in New Zealand (UNICEF 2008). With advances in perinatal and neonatal care strategies, more babies with complex life limiting conditions are surviving for longer periods of time. While palliative care is an established pathway for adults and children with life threatening or life limiting illness, the same is not true for newborn infants (Kain 2006). There is a clear need for a comprehensive neonatal palliative care strategy to support these dying babies and their families at the earliest possible time frame. The purpose of this article is to review the existing scenario in New Zealand and to discuss the issues involved in the organization of a comprehensive neonatal palliative care strategy.

\section{SEARCH STRATEGY}

Medline, PubMed, CinAhL plus and Psychinfo databases were searched using the following key words 1) Newborn infant 2) Palliative care 3) Neonatal palliative care 4) Neonatal End of life care 5) Physicians/doctors 6) Organization. 
The articles obtained were further screened and analyzed to understand the state of neonatal palliative care with a particular reference to New Zealand.

\section{THE NEW ZEALAND SCENARIO}

The Ministry of Health (мон) in New Zealand devised a Palliative Care Strategy in 2001 (MOH 2001) in which the need to develop a national paediatric palliative program was emphasized. Following this, the Paediatric palliative care specialty was established in the Auckland region. Despite this development, childhood palliative care services have lagged behind adult services (Naylor 2010). While the specialty has been set up in the Auckland region, most other district health boards (DHBS) ${ }^{1}$ have not appointed their own palliative care coordinators for children (Drake 2007). The palliative care specialty service in the Auckland region is currently attempting to serve the needs of the region and of other DHBs through protocol development and remote consultations.

The Perinatal Society of Australia and New Zealand (psanz) has developed an 'end-of-life care strategy for children' which provides best practice guidelines in palliative care issues relating to children and new born babies (Drake 2008). A recent study looking at the uptake of this strategy among neonatal practitioners found that only $42 \%$ of clinicians were aware of such a guideline and only $19 \%$ had received any form of training across Australia and New Zealand (Flenady 2010).

The situation is no different in other parts of the world. In America, Catlin and Carter (2001) created a 'neonatal end-of-life protocol' in 2001. But according to many commentators neonatal palliative care remains, at best, ad hoc (Kain 2006, Maginnes 2002, Glicken 2002). Kain goes on to research the reasons why neonatal palliative care has not taken off despite the need and the recognition by various professional bodies. Various barriers to provide palliative care were identified in her study ranging from ethical, moral, and attitudinal apart from resource constraints (Kain 2006).

\section{ATTITUDES OF PHYSICIANS, NURSES AND FAMILIES}

The primary issue identified in the literature as a barrier to neonatal palliative care is the attitude of professionals and parents. Palliative care is an evolving field in a neonatal intensive care (NICU) environment. Despite the prominent presence of paediatric palliative care programs in many institutions over a number of years, neonatal care-givers have been reluctant to embrace the ser- 
vices. This is attributed to the perception of palliation as being the antithesis of neonatal intensive care where the focus is on saving and prolonging lives (Catlin \& Carter 2002). This perception is borne out of a misconception regarding the meaning of palliative care. Catlin and Carter define palliative care as 'a holistic, extensive, family centred care for an infant who has a life threatening/ life limiting illness' (Catlin \& Carter, 2002). Palliative care is not just the care of the 'dying' but it is also the care of the 'living' who are not getting better. The difficulty for the care-giver is to realise that moving towards palliative care is not a failure of curative treatment or withdrawal of care, but is just a switching of care pathway with a different end point, ie. comfort (Maginnes 2002). This change in mindsets is happening in the NICU environment across the world at varying pace (Glicken 2002).

Parents, physicians and nurses seem to approach the concept of palliative care differently (Hefferman 1999). Epstein (2008) explored the differing attitudes of physicians and nurses in end-of-life (EOL) situations and found that the physician group seem to be involved strongly in the decision making with respect to EOL care. The nursing group, conversely, focussed on the actual EOL care experience. Physicians tended to be less involved in EOL care compared to nurses. Paediatric trainees who were surveyed in another study showed a feeling of inadequacy in EOL situations (Boss, 2009) indicating their difficulty of transition to palliative care. However, the literature pertaining to physician attitude towards EOL care is scant (Epstein 2008).

Another study, the Euronic study, a large multi centre trial across 11 countries and 123 neonatal units in Europe set out to determine physician attitudes in EOL situations (McHaffie 1999). The study outlined the physician approach towards the timing of withdrawal of care, the legal and moral frameworks across Europe but did not discuss the state of neonatal palliative care explicitly in the same contexts (Cuttini 2009, McHaffie 1999).

Barr examined Australasian Neonatologists' individual fear of death and the relationship of this attitude towards certain aspects of their EOL decision making (Barr 2007). He reports that physicians with a greater fear of the dying process and of premature death are more likely to be open to the idea of 'death being hastened' with sedation. This study again illustrates the ad hoc nature and the need for a more structured approach to concepts of neonatal palliative care. The situation is gradually changing with more physicians discussing what constitutes a 'good death' and thinking about the memories of families in the context of newborn infant's EOL situation (Epstein 2008). 
Kain summarises the literature with regards to various aspects of nurses' attitude toward EOL care; ranging from moral distress, emotional control, protection, and nurses' grief (Kain 2006). The nurses were shown to think of the palliative care option ahead of physicians, and were shown to encourage physicians to discuss the option with parents (Epstein 2008).

Parental perception of the care of infants with life limiting conditions is a reflection of their culture, religious beliefs, and the norms and opinions of the wider society in which they live (McHaffie 2001). Parental goals may be entirely different from physician's goals. In a study about parental attitudes towards delivery room resuscitation of extremely low birth weight babies, Boss reported that parents did not use the physician-provided morbidity and mortality data to make a decision. Instead they placed religion, spirituality and hope as central to their decision making processes (Boss 2008). This suggests a 'disconnect' between the attitudes of parents and physicians (Levine 1999, Crawley 2002, Poncet 2007).

Societal approaches to dying in general contribute to the slow progress in integrating palliative care into curative care as a dual model. There is a societal impression that advancing technology is a panacea for all ailments on the one hand, and a reliance on hope and miracles on the other. Reluctance to transition to palliative care may stem from these impressions.

Language is part of the societal approach and Hylton-Rushton and Catlin (2002) argue that the absence of a word in the English language to describe a childless parent, similar to the word 'orphan' that describes a parentless child, exemplifies the societal lack of acceptance that children could die. Absence of a dedicated word is a major impediment to palliative care (Hylton-Rushton, 2002). This has been quoted by Kain in her review of the barriers to neonatal palliative care (Kain 2006). One way to address many of the barriers which have been discussed here is through education and training. Communication is also a core issue to address in trying to resolve barriers that involve key multidisciplinary personnel and teams in palliative care.

\section{EDUCATION, TRAINING AND COMMUNICATION}

Society's reluctance to face up to neonatal deaths, and hence palliative care, can be effectively addressed through education, training, communication and understanding. There are many times in a NICU environment when one has to deal with babies on the border zone of viability, and babies who are receiving intensive care despite having life limiting illness (MacDonald 2002). Most of 
the decisions around the transition from curative to palliative care are taken by clinicians with limited formal training in palliative care or ethics (Epstein 2008). In a recent survey of fellows training to be neonatologists, it was found that more than $40 \%$ felt that there was inadequate training in communication and collaborative decision-making processes with families (Boss 2009). Trainee neonatologists felt ill-equipped to discuss the concept of palliative care, spiritual and religious needs of the families in their care. Surprisingly, $25 \%$ felt that there was no need to discuss spiritual or religious needs (Boss 2009). Whilst literature shows that families use spirituality to make decisions, they may not be averse to discussing these aspects with their physician and it has been clearly shown that palliative care can be taught to medical students and trainee physicians with good results (Schiffman 2008, Ross 2005, Han 2005, Bagatell 2002).

Early involvement of the palliative care team as a concept in care would benefit families and practitioners involved in care (Leuthner 2004). Palliative care is not merely about care of the dying but is also about providing holistic care to the infant and to the family from the time of intensive care or learning of the life limiting nature of an illness (Catlin \& Carter 2002). Catlin and Carter make an argument for certain groups of infants receiving both curative care and palliative care from the 'word go' and when the time comes to make a definitive shift towards palliative care. With this in mind, "health professionals especially doctors and nurses should be adequately trained in communicating with patients and their family about the goals of care' (Ministry of Health 2001) and this should include neonatal palliative care. Significantly General Practitioners, midwives and practice nurses could be included in this education strategy.

With advancing neonatology and perinatology, increasing numbers of babies with complex conditions are diagnosed during the antenatal period, and a number of babies survive for varying lengths of time with or without curative treatment. The antenatal period is a good time to introduce the parallel care idea when discussion about possible complications arises. The idea of comfortgoal-directed care needs to be introduced quite early during discussions in the antenatal period and using an interdisciplinary approach. There is considerable reluctance to introduce palliative care at this stage as there is a perception by the medical team and parents that once palliative care is discussed the hope for a cure is being shut out (Baergen 2006). This is a myth about palliative care. Palliative specialists and staff trained in palliative care would emphasise that within palliative care you 'hope for the best while preparing for the worst' (Back 2003). These subtleties of palliative care need to be understood by the 
medical team, and communicated to the family in a clear and effective way. The newer models of palliative care involve considerable overlap of curative and palliative care pathways (Carter \& Bhatia 2001), placing the family back in the centre stage, improving communication, and focusing on symptom management with improved satisfaction of the families as a result.

\section{PALLIATIVE CARE POLICY}

The next core issue in the organisation of palliative care is to have a clear written Neonatal Palliative Care policy. There are national and international policies on delivery of palliative care to neonates, however the delivery of palliative care in a NICU environment remains ad hoc (Maginnes 2002). In New Zealand there is a National Palliative Care strategy (мон 2001), a Paediatric Palliative Care policy as outlined in the Perinatal Society of Australia and New Zealand (PSANZ) policy (Drake 2008), and a broad guideline for the neonatal context is given in the PSANZ policy. There is currently no comprehensive Neonatal Palliative Care policy.

A Paediatric Palliative Care specialist is available in the Auckland region, but there is no adequate support for the rest of the country. Most DHBs do not have trained Paediatric Palliative Care personnel or any regional policy to fall back on. This gap represents a barrier to effective Paediatric and Neonatal Palliative Care which needs to be addressed at the policy level.

The important barriers identified to delivery of effective neonatal palliative care are:

1) the absence of evidence based policy and protocol structure (Brennan, 2008)

2) a lack of trained staff, and

3) the absence of physical space to deliver appropriate palliative care (Kain 2006).

The development of a multi-disciplinary evidence-based policy would result in proper resource planning and allocation, support for physicians and families in providing appropriate neonatal palliative care, resolve conflicts between care givers and families, and ensure a consistent delivery of palliative care.

The lack of a clear neonatal palliative care policy is in essence limiting the provision of such palliative care to these dying babies (Brennan 2008). The development of a local unit based policy based on the PSANZ`s guidelines will 
generate discussions and help to educate staff about the aspects of palliative care and the dialogues which surround its implementation. Consequentially, continuous updating of such policies will keep the debate going so the decisions made in such circumstances would be evidence based and consistent, reflecting the culture and ethos of the multidisciplinary unit rather than that of the individual clinician caring for the baby.

Another advantage of a written policy would be to have a defined list of conditions and situations where palliative care could be considered in parallel with curative treatment. This would facilitate expert involvement from all aspects early on and help to reduce conflicts between professionals and families. Most conflicts between physicians, nurses and parent groups are result from misconceptions about the nature of palliative care. Recent thinking in terms of overlapping care models, where an urgent binding choice to either pathway need not be made straightaway, will reduce the cause for conflicts. This again begs the need for trained personnel at a specialist level and a considered policy for neonatal palliative care.

\section{CULTURAL ISSUES}

Another key issue identified by the NZ palliative care strategy as a major barrier to proper delivery of palliative care is the cultural barrier (мон New Zealand 2001). New Zealand, a historically bi-cultural society, is transforming into a multicultural society which makes the delivery of palliative care more complex. The death of a family member is an emotional time when a community's cultural sensitivities come to the fore and need to be catered to with utmost respect. Cultural competence has always been emphasized in New Zealand; however this still needs to be addressed proactively in a palliative care setting.

Maori people have a different model for health that is more holistic and includes physical, emotional, spiritual and family components. Palliative care personnel would improve the acceptance of their services by acknowledging Maori belief systems while delivering palliative care to babies (Muircroft 2010). There appears to be less awareness about palliative care among some ethnic migrant groups like Pacific and Asian people (Naylor 2010) Most immigrant and Asian families are reluctant to ask for help from health professionals in a death and dying context (Doorenbos 2003).

Spiritual and religious needs in a palliative care context of major ethnicities need to be studied in order to cater for these needs in an effective manner. The Palliative Care Council of New Zealand has reviewed the functioning of the 
Ministry of Health's Strategy (2001) in the context of cancer patients, a group that receives the bulk of palliative care (Naylor 2010). The review notes that the issue of access for Maori and Pacific people is still a cause of concern in New Zealand. Its findings include late referrals, problems with service delivery and issues with cultural competence of workforce. Addressing such issues would help to improve and restructure services. More research into cultural attitudes towards death and dying of various communities would help in the training and sensitisation of staff providing palliative care.

\section{DELIVERY MODELS}

Home/Hospice based versus Hospital based

Palliative care services have been either hospital or home based. There are pros and cons for both models. Neonatal intensive care patients have more often than not utilised hospital based palliative services. There is a general feeling that most parents of NICU babies would opt to remain in the hospital considering the stability of the babies (Bhatia 2006), although this has never been studied in a systematic manner. However once parents were able to overcome the initial resistance towards home based care, families seem to prefer this model (Lopez 2007). A contrary argument is that modern hospitals are more family centred and so parents can comfortably focus on spending time with the baby in the security of a hospital environment (Howe 2007). Rowse argues that home-based palliative care programs can fulfil the requirements of patient and community satisfaction just like hospital-based programs (Rowse 2006).

In Brazil there is currently a paradigm shift in neonatal care from hospital to home, which includes palliative care, that is being favourably reviewed (Lopez 2007). Home-based palliative care programs, with the help of either hospital based specialists or under the guidance of regional hospices, relieve considerable stress on hospital resources, and provide families with the option of being in a friendly and familiar environment at their times of distress. At home, parents are able to function as parents free from hospital rules and regulations, and siblings can also participate in the lives of the newborn and build memories with them (Cavaliere 2007). To be successful the palliative care service needs good and comprehensive home care services, and to provide district health nurses who are supported by the hospital paediatric palliative care team. Hospices have a pre-emptive role to play here.

Hospices can play a significant role by offering home-hospice services. Hospices may be able to provide the appropriate environment and guidance to 
the family of a dying baby. In New Zealand there are no hospices for children and most hospices do not have staff with paediatric or neonatal experience. Even so, hospice staff may be able to provide guidance to the family, the GP and the home care team in managing issues involved in the palliative care of babies, along with the tertiary team. An integrated partnership model between hospice (Arohanui Hospice ${ }^{2}$ ), GP groups, and Mid-Central DHB, exists in the Palmerston North area for the provision of adult palliative care (Stewart 2006). The hospice palliative care coordinator and the GP team works together to deliver effective palliative care. Similar models may be worked out for neonatal palliative care delivery.

Funding for these services will be enabled by mandatory regional recognition of such a requirement in a policy. Existing data from adult and paediatric services could also be used to argue the case for funding the employment of trained personnel in hospices (Brumley 2003). More studies to determine the number of babies (using well defined criteria from international studies and from paediatric programs) who could have utilised palliative care services will provide data to help plan the service. Neonatal care services may have to be predominantly hospital-based initially, with slow movement towards homebased services.

In the hospitals adequate space in neonatal units to deliver EOL care to the baby and support the wider family is crucial especially if the multidisciplinary team of curative and palliative specialists are to work together. At the Dнв level, policy formulations need to address these resource constraints. A home Neonatal Palliative Care service along the lines of the Arohanui Hospice/Mid-Central DHB partnership model (Stewart 2006) could be set up as an integrated arm of the existing paediatric service, with the latter providing guidance at a tertiary level. This would entail sub-regional palliative care coordinators with neonatal experience delivering care alongside district nurses, home care services, general practitioners and hospices. More paediatric/neonatal nurses need to be trained in aspects of palliative care to help this process by populating the various delivery arms including the hospices. In addition, GPs and midwives will also need to be brought on board, and be educated and remunerated adequately for the roles they can play in home-based neonatal palliative care.

\section{Perinatal hospices}

The importance of discussing and involving palliative care personnel as early as possible during the ante-natal period has been discussed earlier as a paral- 
lel strategy when discussion of complications arises. Some of these situations arise when babies at risk of dying are identified during the antenatal period. The concept of a perinatal hospice has come into being in America, where about 30-40 such institutions are catering to many pregnant mothers and their babies who are 'born to die' (Banerjee 2007). The hospice supports the families who decide to go through with the pregnancy despite knowing about the life threatening condition so that a personhood can be committed to the foetus and quality time could be spent with the baby, however short. The concept is all about helping families create memories and cope with loss (Leuthner 2007). There are some criticisms regarding the religious or political connotation of such hospices, especially in the United States, but there seem to be many secular institutions who are acknowledging such needs (Leuthner 2007). A comprehensive study regarding bereavement and the need for a bereavement programme or a perinatal hospice could be undertaken in the New Zealand/ Australasian context for future planning. Charitable organisations like sANDS (Still Birth and Newborn Death Support ${ }^{3}$ ) New Zealand providing support to parents with still birth, abortions and neonatal deaths, may be supported to plan a more comprehensive perinatal palliative care programme.

\section{FUNDING}

Funding is a key issue for a new speciality service like neonatal palliative care. While palliative care services are funded and utilised primarily by adults and children with cancer related illnesses, funding for palliative care of a young infant for non-cancer illness appears to get lower priority (Мон 2001). Funding for palliative care services is a major problem, not only in New Zealand, but internationally across most nations (MacLeod 2002). An organised Neonatal Palliative Care service would reduce the in-patient time and also repeated acute admissions, thereby reducing the costs attached to hospital admissions. Another outcome would be increased consumer satisfaction as a direct result of improved communication from the service. These outcomes would be good reasons to make a case for funding a new service. Additionally field studies to show the need for and utilization of such a service would be a good strategy to recommend.

Palliative care services need to work closely with primary health care in order to retain funding (Kaasa 2007). Newer Palliative Care partnership models such as in Palmerston North, may have to be studied further in order to be replicated nationwide (Stewart 2006, McKinlay 2007). In the New Zealand context the regional neonatal units should come up with their own palliative care policies to justify the funding that is required. 
With the improvement of neonatal care more babies are surviving and living with complex health conditions. Many adults with such complex health care needs are accessing palliative care services already. Unfortunately most neonates and young infants are denied such access due to various barriers, which include lack of awareness, and/or the absence of clear guidelines, resulting in poor needs assessments (Kain 2006). Funding is required for training paediatric, neonatal and public health nurses and clinicians, and for remunerating them adequately. This can only be done through a well thought out policy at a national and regional level.

\section{RECOMMENDATIONS}

The recommendations for New Zealand would be:

1. To develop a policy which gives a list of neonatal scenarios where palliative care may be appropriate for consideration, and details of how to provide effective neonatal palliative care.

2. To reorganise neonatal intensive care in such a way that comfort and curative care is instituted for a certain subset of babies from the beginning.

3. To have staff trained/conversant in palliative care involved in the decision making process quite early in the discussions;

4. To provide for the education and training needs of physicians, GPs and nurses in aspects of palliative care, decision making in EOL situations, and communication skills.

5. To integrate cultural competence teaching into education programmes for physicians, GPs and nurses engaged in Neonatal Palliative care.

6. To formulate a comprehensive service directed by policy and providing home based neonatal palliative care as an option in collaboration with the paediatric Palliative Care service.

\section{CONCLUSIONS}

There is clearly a need for a comprehensive neonatal palliative care strategy. The bottom line is the requirement for a change in attitude towards palliative care. This change will occur only with education and awareness of all stakeholders i.e. professionals and parents. Further research needs to be under- 
taken for needs assessment and service planning. Cultural competence is to be promoted by proactively identifying at risk groups and understanding their spiritual and religious needs. More research into parents' attitude towards bereavement, loss and the need for perinatal hospice support will help develop the service in the future. Research, policy formulation and raising the profile in the public arena will help with funding the service. Hospices need to be supported to become more child and newborn friendly. Paediatric palliative care programs in the country should provide the lead to the NICU program, but given the resource constraints the impetus has to come from within the Neonatal specialty. The understanding that palliation and curative pathways are not mutually exclusive and can be considered as parallel and overlapping concepts is central to the way forward.

\section{NOTES}

1 District Health Boards are responsible for providing or funding healthcare and disability services for their districts. There are 20 DHBs in New Zealand.

2 Arohanui hospice is a specialist palliative care hospice in Mid-Central district, New Zealand providing services for cancer and non-cancer life limiting illness.

3 SANDS New Zealand is a network of parent run organization supporting parents who have experienced death of a baby.

\section{REFERENCES}

Baergen, R. 2006 'How hopeful is too hopeful? Responding to unreasonably optimistic parents', Paediatric Nurse, 32(5):485-486.

Back, A.L., Arnold, R.M. and Quill, T.E. 2003 'Hope for the best and prepare for the worst', Annals of Internal Medicine, 138(5):439-443.

Bagatell, R., Meyer R., Herron S., Berger A. and Villar R. 2002 'When children die: a seminar series for pediatric residents', Pediatrics, 110 (2, pt 1):348-353.

Banarjee, N. 2007 'A place to turn when a newborn is fated to die'. Extracted from http://www.nytimes.com/2007/03/13/health/13hospice.html?_ $\mathrm{r}=1$ \&ref=hospicecare, accessed 22/02/2011.

Barr, P. 2007 'Relationship of neonatologists' end-of-life decisions to their personal fear of death', Archives of Disease in Childhood-Fetal and Neonatal 
Edition, 92(2): F104-F7.

Bhatia, J. 2006 Palliative care in the fetus and newborn, Journal of Perinatology, 26: s24-26.

Boss, R.D., Hutton, N., Sulpar, L.J., West, A.M. and Donohue, P.K. 2008 'Values parents apply to decision-making regarding delivery room resuscitation for high-risk newborns', Pediatrics, 122(3): 583-589.

Boss, R.D., Hutton, N., Donohue, P.K. and Arnold, R.M. 2009 'Neonatologist Training to Guide Family Decision Making for Critically Ill Infants', Archives of Paediatrics and Adolescent Medicine, Volume (9):783-8.

Brennan, F., Gwyther, L. and Harding, R. 2008 'Palliative Care as a Human Right'. Extracted from http://www.soros.org/initiatives/health/focus/ipci/ articles_publications/publications/pchumanright_20080101/pchumanright_20080101.pdf, accessed 10/03/2011.

Brumley, R.D., Enguidanos, S. and Cherin D.A. 2003 Effectiveness of a home based palliative care program for end of life, Journal of Palliative Medicine, 6(5): $715-24$.

Carter, B.S. and Bhatia, J. 2001 Comfort/palliative care guidelines for neonatal practice: development and implementation in an academic medical centre, Journal of Perinatology, 21(5): 279-283.

Catlin, A. and Carter, B.S. 2001 'Creation of a neonatal end-of-life palliative-care protocol', Journal of Clinical Ethics, 12(3):316-8.

Catlin, A, and Carter, B.S. 2002 'State of the art: Creation of a neonatal end-of-life palliative care protocol'. Journal of Perinatology, 22(3): 184-195

Cavaliere, T. and Cavaliere, T. 2007 'Should neonatal palliative care take place at home, rather than the hospital? Pro', MCN, American Journal of Maternal Child Nursing, 5:270.

Crawley, L.M., Marshall, P.A., Lo, B. and Koenig, B.A. 2002 'End-of-Life Care Consensus Panel Strategies for culturally effective end-of-life care', Annals of Internal Medicine, 136(9): 673-679.

Cuttini, M., Casotto, V., de Vonderweid, U., Garel, M., Kollee, et al. 2009 'Neona- 
tal end-of-life decisions and bioethical perspectives', Early Human Development, 85(10 Suppl): s21-5.

Doorenbos, A. 2003 'Hospice access for Asian Indian immigrants', Journal of Hospice and Palliative Nursing, 5(1):27-33.

Drake, R. 2007 Feedback on Draft National Specialist Palliative Care Service Specifications. Wellington: The Paediatric Society of New Zealand Extracted from http://www.paediatrics.org.nz/files/positionstatements/consultationNationalSpecialistPalliativeCareServiceSpecs(1)Draft612Feb.doc, accessed 22/02/2011.

Drake, R., Ball, E., Cheng, N., Gillis, J., Phillips, N., et al. 2008 Decision making at the End of Life in Infants, Children and Adolescents. A Policy of the Paediatrics \& Child Health Division of The Royal Australasian College of Physicians. Extracted from http://www.racp.edu.au/download. cfm? downloadfile $=$ B5603385-D3A3-F3B4-7159013AE33D4697\&typename $=\mathrm{dmFile} \&$ fieldname $=$ filename, accessed 24/02/2011.

Epstein, E.G. 2008 'End-of-life experiences of nurses and physicians in the newborn intensive care unit', Journal of Perinatology, 28(11):771-8.

Flenady, V., Mahomed, K., Ellwood, D., Charles, A., Teale, G., et al. 2010 Uptake of the Perinatal Society of Australia and New Zealand perinatal mortality audit guideline, Australian and New Zealand Journal of Obstetrics and Gynaecology, 50(2):138-43.

Glicken, A.D., and Merenstein, G.B. 2002 'A neonatal end-of-life palliative protocol - an evolving new standard of care?' Neonatal Network, 21(4):35-36.

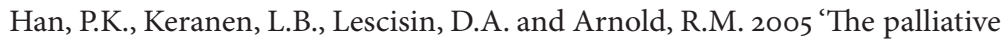
care clinical evaluation exercise (CEX): an experience-based intervention for teaching end-of-life communication skills', Academic Medicine, 8o(7):669676.

Hefferman, P., and Heilig, S. 1999 'Giving "moral distress” a voice: ethical concerns among neonatal intensive care unit personnel', Cambridge Quarterly of Healthcare Ethics, 8(2):173-178.

Howe, T.H. and Howe, T.-H. 2007 'Should neonatal palliative care take place at home, rather than the hospital? Con', MCN, American Journal of Maternal 
Child Nursing, 32(5): 271.

Hylton-Rushton, C. and Catlin, A. 2002 'Pediatric palliative care: the time is now!' Pediatric Nursing, 28(1):57-60.

Kain, V.J. 2006 'Palliative care delivery in the NICU: What barriers do neonatal nurses face?' Neonatal Network, 25(6):387-392.

Kaasa, S., Jordhøy, M.S. and Haugen, D.F. 2007 'Palliative care in Norway: a national public health model', Journal of Pain and Symptom Management, 33(5):599-604.

Leuthner, S.R. and Pierucci, R. 2001 'Experience with Neonatal palliative care consultation at the medical college of Wisconsin - Children Hospital of Wisconsin', Journal of Palliative Medicine, 4(1):39-47.

Leuthner, S., Jones, E.L., Leuthner, S. and Jones, E.L. 2007. 'Fetal Concerns Program: a model for perinatal palliative care', MCN, American Journal of Maternal Child Nursing, 32(5):272-8.

Levine, C., Zuckerman, C. 1999 'The trouble with families: toward an ethic of accommodation', Annals of Internal Medicine, 130(2):148-152.

Lopes, T.C., Mota J.A.C. and Coelho, S. 2007 Perspectives from a home based neonatal care program in Brazil's single health system, Rev Latino-am Enfermagem, 2007 julho-agosto; 15(4):543-48.

MacDonald, H. 2002 'Perinatal care at the threshold of viability', Pediatrics, 110(5):1024-1027.

MacLeod, R.D. 2002 Caring for children who are dying (editorial), New Zealand Medical Journal, 115(1163): U195.

Maginnes, E. 2002 'Palliative care in the neonatal population', Neonatal Network, $21(4): 77-78$.

McHaffie, H.E., Cuttini, M., Brölz-Voit, G., Randag, L., Mousty, R., et al. 1999 'Withholding/withdrawing treatment from neonates: legislation and official guidelines across Europe', Journal of Medical Ethics, 25(6): 440-446.

McHaffie, H., Lyon, A., and Hume, R. 2001 'Deciding on treatment for neonates: 
the parents' perspective', European Journal of Paediatrics, 16o(6):339-344

McKinlay, E. and McBain, L. 2007 'Evaluation of the Palliative Care Partnership: a New Zealand solution to the provision of integrated palliative care', New Zealand Medical Journal, 12:120(1263): U2745.

Muircroft, W.M., McKimm J., William L. and Macleod R.D. 2010 'A New Zealand perspective on palliative care for Maori', Journal of Palliative Care, 26(1):54-8.

Naylor, W. 2010 Positioning Palliative Care in New Zealand: A review of government health policy in relation to the provision of Palliative Care Services in New Zealand. Extracted from http://cancercontrolnz.govt.nz/files/Positioning\%2oPalliative\%2ocare\%20in\%2onZ.pdf, accessed 22/02/2011.

Ministry of Health. 2001 New Zealand Palliative Care Strategy. Extracted from http://www.MOH.govt.nz/MOH.nsf/o/65C53A08E9801444CC256E62000AA $\mathrm{D} 8 \mathrm{o} /$ \$File/palliativecarestrategy.pdf, accessed 22/02/2011.

Poncet, M.C., Toullic, P., Papazian, L., et al. 2007 'Burnout syndrome in critical care nursing staff', American Journal of Respiratory and Critical Care Medicine, 75(7): 698-704.

Ross, D.D., Shpritz, D., Hull M.M. and Goloubeva, O. 2005 'Long-term evaluation of required coursework in palliative and end-of-life care for medical students', Journal of Palliative Medicine, 8(5):962-974.

Rowse, V. 2006 Home based palliative care for children: the case for funding, Paediatric Nursing, 18(7): 20-4.

SANDS NZ. Extracted from http://www.sands.org.nz/ accessed 22/02/2011.

Schiffman, J.D., Chamberlain, L.J., Palmer, L., Contro, N., Sourkes, B. and Sectish, T.C. 2008 'Introduction of a pediatric palliative care curriculum for pediatric residents', Journal of Palliative Medicine, 11(2):164-170.

Stewart, B., Allan, S., Keane, B., Marshall, B., Ayling, J. et al. 2006 Palliative Care Partnership: a successful model of primary/secondary integration, New Zealand Medical Journal, 119(1242). Extracted from http://www.nzma.org. nz/journal/119-1242/2235/ accessed 22/o2/2011. 
UNICEF, 2008 Extracted from http://www.unicef.org/infobycountry/newzealand_ statistics.html accessed 22/02/2011.

wHO, 1998 Palliative care, Extracted from http://www.who.int/cancer/palliative/ definition/en/ accessed 22/02/2011. 